\title{
Biomechanical Modeling of Posterior Instrumentation of the Scoliotic Spine
}

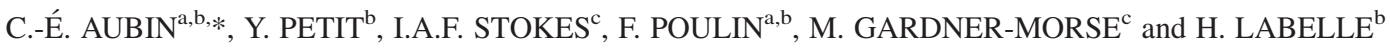

${ }^{a}$ Department of Mechanical Engineering, École Polytechnique, P.O. Box 6079, Station “Centre-ville”, Montréal, Quebec, Canada H3C 3A7; ${ }^{\mathrm{b}}$ Research Center, Sainte-Justine Hospital, Mother-Child University Hospital, University of Montreal, 3175, Côte Sainte-Catherine Rd, Montréal, Quebec, Canada H3T 1C5; ' Department of Orthopedics and Rehabilitation, University of Vermont, Stafford Hall, Burlington, VT 05405-0084, USA

\begin{abstract}
Scoliosis is a three-dimensional deformation of the spine that can be treated by vertebral fusion using surgical instrumentation. However, the optimal configuration of instrumentation remains controversial. Simulating the surgical maneuvers with personalized biomechanical models may provide an analytical tool to determine instrumentation configuration during the pre-operative planning. Finite element models used in surgical simulations display convergence difficulties as a result of discontinuities and stiffness differences between elements. A kinetic model using flexible mechanisms has been developed to address this problem, and this study presents its use in the simulation of Cotrel-Dubousset Horizon surgical maneuvers. The model of the spine is composed of rigid bodies corresponding to the thoracic and lumbar vertebrae, and flexible elements representing the intervertebral structures. The model was personalized to the geometry of three scoliotic patients (with a thoracic Cobb angle of $45^{\circ}, 49^{\circ}$ and $39^{\circ}$ ). Binary joints and kinematic constraints were used to represent the rod-implant-vertebra joints. The correction procedure was simulated using three steps: (1) Translation of hooks and screws on the first rod; (2) $90^{\circ}$ rod rotation; (3) Hooks and screws look-up on the rod. After the simulation, slight differences of $0-6^{\circ}$ were found for the thoracic spine scoliosis and the kyphosis, and of $1-8^{\circ}$ for the axial rotation of the apical vertebra and for the orientation of the plane of maximum deformity, compared to the real post-operative shape of the patient. Reaction loads at the vertebra-implant link were mostly below $1000 \mathrm{~N}$, while reaction loads at the boundary conditions (representing the overall action of the surgeon) were in the range $7-470 \mathrm{~N}$ and maximum torque applied to the rod was $1.8 \mathrm{Nm}$. This kinetic modeling approach using flexible mechanisms provided a realistic representation of the surgical maneuvers. It may offer a tool to predict spinal geometry correction and assist in the preoperative planning of surgical instrumentation of the scoliotic spine.
\end{abstract}

Keywords: Biomechanical model; Scoliosis; Spinal instrumentation; Multi-body model; Computer assisted surgery

\section{INTRODUCTION}

Scoliosis is a three-dimensional (3D) deformation of the spine. Severe cases usually are treated by spinal instrumentation and osteosynthesis, which allow curvature correction and post-operative stabilization of the trunk [1]. Typical of the current instrumentation is the CotrelDubousset system [2] that relies on combined rotation and distraction forces to achieve an appropriate 3D correction, using a deformed rod on the concave side of the spine secured to the spine by hooks and screws [3]. A torque is applied to rotate the rod and realign the curved spine towards the sagittal plane. The hooks and screws are fixed on the rod and, finally, a second rod and devices for transverse traction are inserted to increase system stability and stiffness. Planning the surgical technique requires the determination of many variables that include the spinal segment to instrument, the number and level of vertebral attachments, the length and curvature of the rods, etc.

Even with the recent advances in spinal correction techniques, the best instrumentation configuration remains controversial $[4,5]$. By predicting surgical correction as a function of the instrumentation variables, numerical modeling can be used to guide surgeons in deciding the best instrumentation configuration and location. In order to achieve this for individual patients, prediction of surgery outcome and biomechanical impact of spinal implants can be obtained by simulating the surgical maneuvers with personalized biomechanical models [6,7]. Biomechanical models based on the non-linear finite

*Corresponding author. Address: Canada Research Chair "CAD Innovations in Orthopedic Engineering", Department of Mechanical Engineering, École Polytechnique, P.O. Box 6079, Station "Centre-ville", Montréal Qué., Canada H3C 3A7. Fax: + 1-514-340-5867.

E-mail: carl-eric.aubin@polymtl.ca 
elements (FE) method have been used to simulate CotrelDubousset instrumentation procedures [8-10]. This mathematical method uses iterative procedures to converge on system equilibrium, and provide force and displacement values for each element. However, because of the discontinuous nature of the system (mechanism composed of several parts and kinematic joints), large variations in displacements and element stiffness, it produces mathematical inconsistencies that cause convergence difficulties [11].

In order to address this problem, the concepts of a kinetic model using flexible mechanisms to represent the functional units of the spine have been proposed [12]. The aim of this paper is to compare the predictions of this modeling approach for the simulation of CotrelDubousset surgical maneuvers with the documented surgical outcome of three patients with idiopathic scoliosis.

\section{METHODS}

The biomechanical model of the spine was developed using a kinetic modeling approach based on the differential equations of dynamics and algebraic equations describing the constraints of the system in the form: $\mathbf{M} \ddot{\mathbf{q}}+$ $\mathbf{K q}+\mathbf{C}^{\mathrm{T}} \boldsymbol{\lambda}=Q_{\mathrm{e}}+Q_{\mathrm{r}}$ where $\mathbf{M}$ is the mass matrix of the rigid bodies, $\mathbf{K}$ is the stiffness matrix of flexible elements, $\mathbf{C}$ are algebraic equations representing the constraints of the system, $Q_{\mathrm{e}}$ and $Q_{\mathrm{r}}$, respectively, are external and reaction forces, $\mathbf{q}$ is the matrix of the generalized coordinates of the elements of the system and $\boldsymbol{\lambda}$ is a vector of the Lagrange coefficients. The basic concepts of this model were presented by Poulin et al. [12], and are summarized here with additional details and recent developments. It was implemented with ADAMS 8.2 software (Mechanical Dynamics Inc., Ann Arbor, USA).
The model of the spine is composed of rigid bodies corresponding to the thoracic and lumbar vertebrae that were visible in the intra-operative radiographs (From T4 to L3 or L2), and flexible elements representing the intervertebral structures. Local (vertebral) coordinate systems were defined at the centroid of each vertebral body. Their location and orientation were obtained from pre-instrumentation radiographs of a scoliotic patient using the 3D multi-view reconstruction method developed by Cheriet et al. [13,14]. A comprehensive geometric representation of each vertebra is obtained using an atlas of detailed vertebrae, deformed by a dual kriging technique [15] to fit the reconstructed points [16]. The global behavior of the intervertebral structures is represented using a massless beam element formulated according to the Timoshenko theory [17], by which reaction forces required to displace one vertebra with respect to the adjacent one are calculated. The 3D beam with $30 \mathrm{~mm}$ posterior offsets proposed by Gardner-Morse and Stokes [8] was used to represent these intervertebral structures. The mass $(M)$ of all rigid bodies was arbitrarily fixed to $0.3 \mathrm{~kg}$ since it has no effect in quasi-static analyses.

In this study, the model was personalized to the geometry of three patients $(16.8,14.6$ and 15.1 year-old girls) with a right thoracic deformity (intra-operative Cobb angles before the instrumentation: $45^{\circ}, 49^{\circ}$ and $39^{\circ}$ ), who were operated with the Cotrel-Dubousset Horizon (CDH) instrumentation (Medtronic Sofamor-Danek, Memphis, $\mathrm{TN}$ ). Three sets of radiographs (two intra-operative, preand post-instrumentation, and one post-operative) were obtained to document surgical correction and assess simulation results (Table I).

The insertion points of the hooks and screws on the left side of the vertebrae as well as the first rod 3D geometry (on concave side) were calculated from the postinstrumentation 3D reconstruction. In order to obtain

TABLE I Geometric indices: pre- and post-instrumentation measures and simulation results for the three patients

\begin{tabular}{|c|c|c|c|c|c|}
\hline & \multirow[b]{2}{*}{ Pre-instrumentation } & \multicolumn{3}{|c|}{ Simulation } & \multirow[b]{2}{*}{ Post-instrumentation } \\
\hline & & Step 1 (translation) & Step 2 (rod rotation) & Step 3 (spring-back) & \\
\hline \multicolumn{6}{|l|}{ Cobb angle $\left({ }^{\circ}\right)$} \\
\hline Patient 1 & 45 & 6 & 15 & 15 & 10 \\
\hline Patient 2 & 49 & 23 & 16 & 16 & 16 \\
\hline Patient 3 & 39 & 25 & 41 & 22 & 28 \\
\hline \multicolumn{6}{|l|}{ Kyphosis $\left({ }^{\circ}\right)$} \\
\hline Patient 1 & 20 & 15 & 32 & 32 & 30 \\
\hline Patient 2 & 18 & 16 & 11 & 12 & 12 \\
\hline Patient 3 & 4 & 7 & 18 & 28 & 34 \\
\hline \multicolumn{6}{|c|}{ Axial rotation $\left({ }^{\circ}\right)$ of the apical vertebra (T9) } \\
\hline Patient 1 & -4 & 0 & -15 & -9 & -6 \\
\hline Patient 2 & 0 & 4 & 3 & 5 & -2 \\
\hline Patient 3 & -5 & -9 & -25 & -9 & -2 \\
\hline \multicolumn{6}{|c|}{ Orientation of the plane of maximum deformity with respect to the sagittal plane $\left(^{\circ}\right)$} \\
\hline Patient 1 & 43 & 127 & 77 & 74 & 82 \\
\hline Patient 2 & 83 & 85 & 17 & 30 & 38 \\
\hline Patient 3 & 78 & 46 & 44 & 44 & 45 \\
\hline
\end{tabular}


realistic behavior of the hooks and screws, generalized constraints in the form $\mathbf{C}^{i}\left(\mathbf{q}^{i}, t\right)=0$ were introduced to restrain appropriate degrees of freedom (DOF) at the connection points with the rod and vertebrae. This was achieved by defining binary joints (revolute, spherical, cylindrical, prismatic and rigid) and kinematic constraints (translation and rotation). For instance, the hook-vertebra and the screw-vertebra joints were defined using revolute joints allowing only 1 DOF (rotation of the implant about the axis of the pedicle to which it is attached). The implant-rod joints were defined as hinge-like connections (cylindrical joints), with 2 DOFs each (along and around the rod curve). The 5-mm diameter rod was considered rigid because its profile was obtained from the already deformed post-operative shape.

Boundary conditions were imposed to represent the behavior of the anesthetized patient on the operating table. All DOFs except frontal plane rotation were fixed at the lowest vertebra in the model (L3 or L2). The fourth thoracic vertebra (highest vertebra) was allowed to translate and rotate freely. The highest implant-rod joint (at T4) was constrained in translation to avoid any rigid displacement between the rod and the spine.

Three simulation steps (Fig. 1) were used to represent the surgical maneuvers:

Step 1: Translation of hooks and screws on the first rod. The initial distance between the rod and hook/screw extremities was brought to zero using translation displacement constraints. Then, translational joints (1 DOF) were defined between each implant and the rod. After translation of all implants to the rod, the cylindrical joints were introduced to connect the implant to the rod.

Step 2: Rotation of the rod. A rotational displacement constraint was gradually applied on the rod until $90^{\circ}$ of rotation about the rod axis defined by the rod's endpoints to reproduce the rod rotation maneuver. As the rod rotated, the implants were free to slide (except at $\mathrm{T} 4)$ and rotate along the rod curve (2 DOFs).

Step 3: Lock-up of the hooks and screws on the rod. Lock-up was produced by coupling all DOFs at the connection point with fixed (rigid) mechanical joints (0 DOF), and by replacing the hook-vertebra revolute joints by spherical joints to allow appropriate reorientation. Finally, the rotational displacement imposed to the rod was released, allowing the spineinstrumentation system to recover a new equilibrium configuration (spring back).

The installation of the second rod and the transverse traction devices was not simulated here because we considered these components to provide increased stiffness to the system without significantly changing the spinal shape. The three steps were applied very slowly
( $t=100 \mathrm{~s}$ each) to ensure that accelerations were negligible.

The following measures of spinal shape were used to compare simulation results with the post-instrumentation radiographs: thoracic Cobb angle (angle between the intersection of two lines perpendicular to the spinal curve at its inflection points), kyphosis (angle between the intersection of two lines perpendicular to the spinal curve at $\mathrm{T} 4$ and $\mathrm{T} 12)$, axial rotation at the apex vertebra [18] and the orientation of the plane of maximum deformity (angle of the vector normal to the plane defined by the apex and the two end-vertebrae, with respect to the sagittal plane) [19]. The distraction force generated between the implants and the rod during the simulation of the translation maneuver as well as the maximum torque applied to the rod during the rod rotation also were calculated.

\section{RESULTS}

The spinal shape was first examined in the coronal, sagittal and transverse planes at all simulation steps. Figure 1 shows the simulation results of the first patient, which are quite similar as the results of the other two patients. An important part of simulated surgical correction occurred in the coronal plane during the translation phase and in the sagittal and transverse planes during the rod rotation phase. For instance, and over-correction of Cobb angle was obtained in the frontal plane after the translation step for the first and third patients $\left(4^{\circ}\right.$ and $\left.3^{\circ}\right)$, and an under-correction of $-7^{\circ}$ was obtained for the second patient (Table I). Spinal correction was lost in the remaining steps for the first patient $\left(9^{\circ}\right)$, but was slightly improved of $7^{\circ}$ and $3^{\circ}$ for the two other cases. Actually, differences of $5^{\circ}, 0^{\circ}$ and $6^{\circ}$ were found with the postinstrumentation Cobb angles (Table I). In the sagittal plane, the correction mostly was achieved at the rod rotation step, with final slight $2^{\circ}, 0^{\circ}$ and $6^{\circ}$ kyphosis differences with the real correction (Table I). In the transverse plane, a large axial rotation was found after the rod rotation maneuver. But this over-rotation was lost after the implants lock-up and the removal of the applied torque. Thus, final differences of $3^{\circ}, 7^{\circ}$ and $6.7^{\circ}$ with the real correction were found for the axial rotation of the apical vertebra after the three steps. There was more scatter in the plane of maximum deformity, which is a regional measure of the effect of the surgical maneuver on the transverse plane projection of the spine. However, its orientation was always predicated in the correct direction with a final difference of, respectively, $8^{\circ}, 8^{\circ}$ and $1^{\circ}$ between the last simulation step and the post-operative configurations for the three patients.

Calculated reaction forces were mostly below $1000 \mathrm{~N}$ (except at two levels for patient 1) at the vertebra-implant links during the surgical phases (Table II). After the translation step, the highest reaction force occurred, respectively, at T12 $(1094 \mathrm{~N}), \mathrm{T} 10(384 \mathrm{~N})$ and T8 $(712 \mathrm{~N})$ 

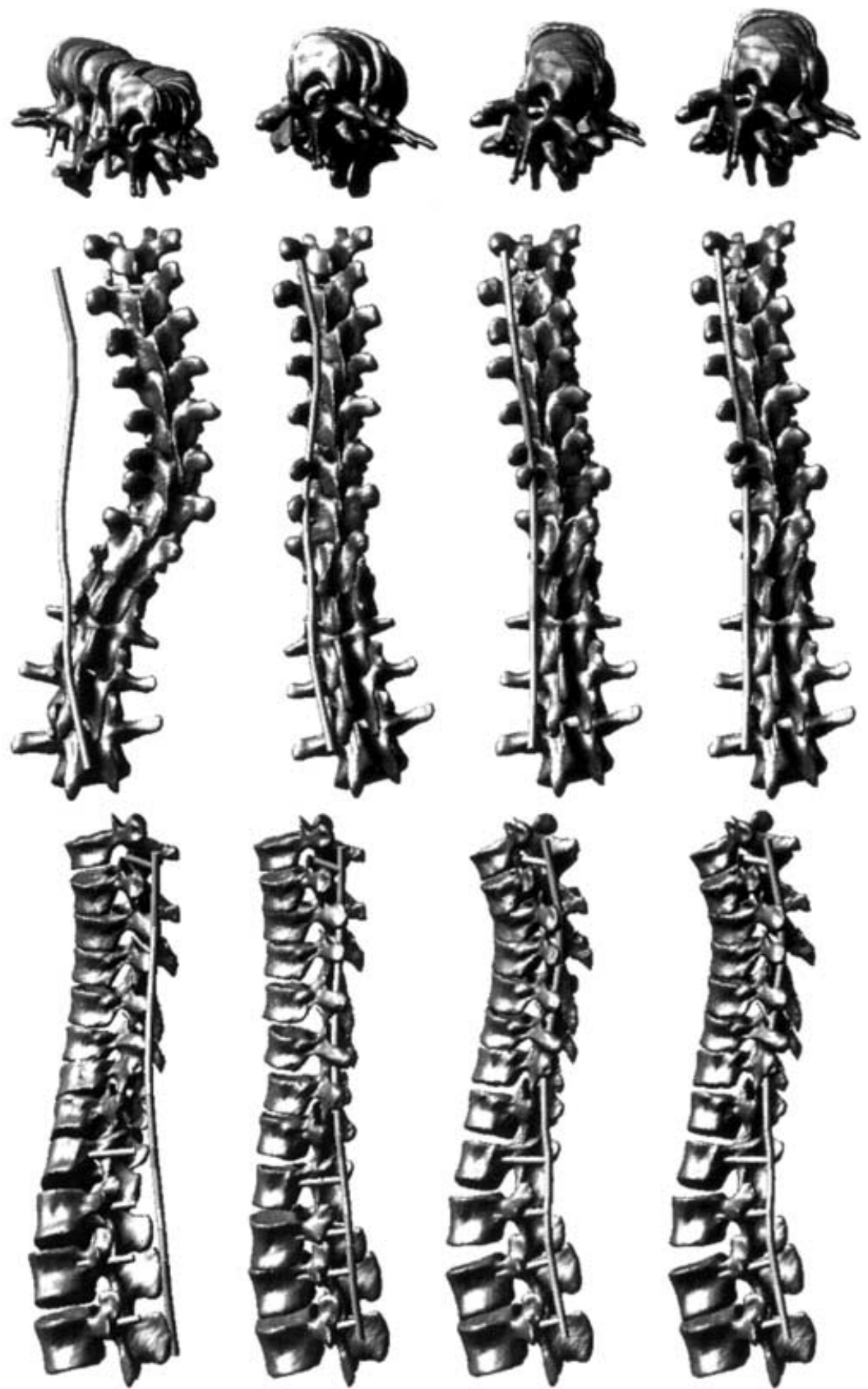

a

c

d

FIGURE 1 Postero-anterior, lateral and transverse plane representation of the first patient's spine and instrumentation during a simulation: (a) initial geometry; (b) after translation of hooks and screws on the first rod; (c) after rotation of the rod; (d) after lock-up of hooks and screws on the rod.

for the three patients, while the lowest reaction force occurred at T6 $(136 \mathrm{~N}), \mathrm{L} 2(71 \mathrm{~N})$ and T10 $(17 \mathrm{~N})$. After the rod rotation step, the highest reaction forces occurred, respectively, at L2 $(1073 \mathrm{~N}), \mathrm{T} 10(319 \mathrm{~N})$ and $\mathrm{T} 8(730 \mathrm{~N})$ and the lowest reaction forces occurred at T6 $(85 \mathrm{~N}), \mathrm{L} 1$ $(32 \mathrm{~N})$ and $\mathrm{L} 1(30 \mathrm{~N})$. After the last step, the highest forces stayed almost constant. These reaction loads seem high, especially for patient 1 . However, the total reaction loads at the boundary conditions (equivalent to the action of the surgeon), respectively, were $73,-7$ and $-470 \mathrm{~N}$ for the three patients after the translation step. During the rod rotation step, maximum torques of $1.3,1.5$ and $1.8 \mathrm{Nm}$, respectively, were applied to the rod to produce the $90^{\circ}$ rotation, and total reaction forces of $89,-22$ and $173 \mathrm{~N}$ were found at the boundary conditions. At the end, the system was equilibrated with no residual load $(<1 \mathrm{~N})$.

\section{DISCUSSION}

The simulations of spinal shape changes agreed well with observed surgical results: gradual correction during the surgical procedures (translation of hooks and screws to the rod and rod rotation) and minor loss after hooks 
TABLE II Reaction forces at the implant-vertebra links for the three patients

\begin{tabular}{|c|c|c|c|c|}
\hline \multirow[b]{2}{*}{ Level } & \multirow[b]{2}{*}{ Implant type } & \multicolumn{3}{|c|}{ Reaction forces $(\mathrm{N})$} \\
\hline & & $\begin{array}{c}\text { Step } 1 \\
\text { (translation) }\end{array}$ & $\begin{array}{c}\text { Step } 2 \\
(\operatorname{rod} \text { rotation) }\end{array}$ & $\begin{array}{c}\text { Step } 3 \\
\text { (spring-back) }\end{array}$ \\
\hline \multicolumn{5}{|c|}{ (Patient 1) } \\
\hline $\mathrm{T} 4$ & Pedicle hook & 414 & 418 & 493 \\
\hline T6 & Pedicle hook & 136 & 85 & -28 \\
\hline $\mathrm{T} 8$ & Pedicle hook & -487 & -482 & -531 \\
\hline $\mathrm{T} 10$ & Pedicle hook & -886 & -659 & -495 \\
\hline $\mathrm{T} 12$ & Pedicle screw & 1094 & 681 & 608 \\
\hline L1 & Pedicle screw & -687 & -620 & -621 \\
\hline $\mathrm{L} 2$ & Pedicle screw & 836 & 1073 & 1071 \\
\hline L3 & Pedicle screw & -347 & -407 & -498 \\
\hline \multicolumn{5}{|c|}{ (Patient 2) } \\
\hline $\mathrm{T} 4$ & Pedicle hook & 187 & 259 & 255 \\
\hline T6 & Pedicle hook & -154 & -288 & -291 \\
\hline $\mathrm{T} 8$ & Pedicle hook & 96 & 145 & 146 \\
\hline $\mathrm{T} 10$ & Pedicle hook & -384 & -319 & -316 \\
\hline L1 & Pedicle screw & -169 & 32 & 42 \\
\hline $\mathrm{L} 2$ & Pedicle screw & 71 & 149 & 163 \\
\hline \multicolumn{5}{|c|}{ (Patient 3) } \\
\hline $\mathrm{T} 4$ & Pedicle hook & 619 & 193 & 193 \\
\hline T6 & Pedicle hook & -311 & 258 & 258 \\
\hline $\mathrm{T} 8$ & Pedicle hook & -712 & -730 & -730 \\
\hline $\mathrm{T} 10$ & Pedicle hook & -17 & -133 & -133 \\
\hline $\mathrm{L} 1$ & Pedicle screw & -97 & -30 & -30 \\
\hline $\mathrm{L} 2$ & Pedicle screw & -279 & -352 & -352 \\
\hline L3 & Pedicle screw & 587 & 621 & 795 \\
\hline
\end{tabular}

and screws lock-up in order to release excessive forces induced by the correction maneuvers and stored in the flexible elements (elastic spring-back). Despite small differences between the intra- and post-operative data in the frontal and sagittal planes, the slightly larger differences in the transverse plane can mostly be attributed to the sensitivity of the measurements and inadequate representation of motion segment behavior during surgery using published data of Stokes and Gardner-Morse $[8,10,19]$, which was not specific to the patients analyzed in this study. A portion of the difference also could be attributed to the approximation of the maneuvers. For instance, since we did not have any accurate values for the rod rotation, we assumed the value of $90^{\circ}$, which is probably close to the true value. The maximum torque measured during the rod rotation maneuver is of the same order as those measured intra-operatively by Duke et al. [21]. Even if plausible total reaction forces were obtained, the maximum force during the translation maneuver is quite high at only a few levels and is near the values reported by Gayet et al. [22] for pedicle screws pull-out. This high level of reaction forces most probably has been caused by over-constrained translational displacements between the rod and the implants or by the rigid rod and may have been avoided by allowing additional DOFs or rod deformable capabilities. This explanation was supported by significant reduction in reaction loads (approx. 12\%) at the level where the maximum reaction was reached (T12) for the first patient when slight changes in the position of the implant relative to the vertebra $(2 \mathrm{~mm})$ were tested.

The complete simulation (including pre- and postprocessing operations) took less than $30 \mathrm{~s}$ in batch mode (no interactive visualization) and $1 \mathrm{~min} 30 \mathrm{~s}$ using interactive graphical visualization on a Pentium III $933 \mathrm{MHz}$ PC with $382 \mathrm{Mb}$ of random access memory and an Nvidia TNT2 display adapter. No pivotal error (singularity) due to discontinuities was found since the model was not over-constrained (the number of DOF equals the number of constraints).

This kinetic modeling approach using flexible mechanisms provided a realistic representation of the surgical maneuvers while reducing the convergence problems associated with non-linearities and stiffness differences that are inherent to finite element approach of discontinuous structures. Also, since this approach required fewer simulations to complete the surgical maneuver sequence, overall computational time was very small. However, the flexible mechanism approach could not provide measurement of internal stresses and deformations in the elements. The three spines are representative of the most frequently occurring scoliotic curves; the study has yet to be applied to correction of less common curve types.

The surgical simulations produced in this study were limited to the maneuvers performed with the patients supported on the operating table and anesthetized. Differences in spinal geometry with the pre- and postoperative standing positions [23] were not considered. Since only post-operative instrumentation measurements were available, it was not possible to use the initial rod shape, and represent its elastic deformation when subjected to the forces generated by the instrumentation. The rigid shape of the rod that was used in these simulations was its final (deformed) shape, so it is considered that the equilibrium forces in this position were correct, providing that no plastic deformation of the rod had occurred.

In conclusion, while FE modeling may prove essential when studying anatomical stress levels during surgery, the study of spinal geometry changes caused by surgery proved to be more practical with kinematic modeling. Thus, due to its simplicity and realism, the flexible mechanisms approach has the potential to assist in the preoperative planning of posterior surgical instrumentation of the scoliotic spine for predicting the outcome of different surgical strategies.

\section{Acknowledgements}

Research funded by the Natural Sciences and Engineering Research Council of Canada and the Canada Research Chair program.

\section{References}

[1] Moe, J.H., Bradford, D.S., Lonstein, J.E., Ogilvie, J.W. and Winter, R.B. (1987) Moe's Textbook of Scoliosis and Other Spinal Deformities, 2nd Ed. (Saunders, Philadelphia, USA) 651. 
[2] Cotrel, Y., Dubousset, J. and Guillaumat, M. (1988) "New universal instrumentation in spinal surgery", Clin. Orthop. 227, 10-23.

[3] Denis, F. (1988) "Cotrel-Dubousset instrumentation in the treatment of idiopathic scoliosis", Orthop. Clin. N. Am. 19, 291-311

[4] Takahashi, S., Delecrin, J. and Passuti, N. (1997) "Changes in the unfused lumbar spine in patients with adolescent idiopathic scoliosis. A 5- to 9-year assessment after Cotrel-Dubousse instrumentation", Spine 22, 517-523.

[5] Vaughan, J.J., Winter, R.B. and Lonstein, J.E. (1996) "Comparion of the use of supine bending and traction radiographs in the selection of the fusion area in adolescent idiopathic scoliosis", J. Spinal Disord. 9, 214-219.

[6] Goel, V.K. and Gilbertson, L.G. (1995) "Applications of the finite element method to thoracolumbar spinal research-past, present and future", Spine 20, 1719-1727.

[7] O’Toole, R.V., Jaramaz, B., DiGioia, A.M., Visnic, C.D. and Reid, R.H. (1995) "Biomechanics for preoperative planning and surgical simulations in orthopaedics", Comput. Biol. Med. 25, 183-191.

[8] Gardner-Morse, M. and Stokes, I.A.F. (1994) "Three-dimensiona simulations of the scoliosis derotation manoeuvre with CotrelDubousset instrumentation”, J. Biomech. 27, 177-181.

[9] Leborgne, P., Skalli, W., Lecire, C., Dubousset, J., Zeller, R. and Lavaste, F. (1999) "Simulations of CD surgery on a personalized finite element model: preliminary results", Proceedings of the IRSSD, 28th June-1st July.

[10] Stokes, I.A.F. and Gardner-Morse, M. (1995) "Simulation of surgical manoeurves with C-D instrumentation", Three-dimensional Analysis of Spinal Deformities (IOS Press, Amsterdam), pp. 377-380.

[11] Stokes, I.A.F., Gardner-Morse, M., Aubin, C.É., Poulin, F and Labelle, H. (1999) "Biomechanical simulations for planning of scoliosis surgery", Three-dimensional Analysis of Spinal Deformities (IOS Press, Amsterdam) Vol. 59, pp. 343-346.

[12] Poulin, F., Aubin, C.É., Stokes, I.A.F., Gardner-Morse, M. and Labelle, H. (1998) "Biomechanical modeling of instrumentation for the scoliotic spine using flexible elements: a feasibility study", Ann Chir. 52(8), 761-767, [in French].
[13] Cheriet, F., Delorme, S., Dansereau, J., Aubin, C.É., de Guise, J.A. and Labelle, H. (1999) "Perioperative radiographic reconstruction of the scoliotic vertebral column", Ann. Chir. 53(8), 808-815, [in French].

[14] Cheriet, F., Dansereau, J., Petit, Y., Aubin, C.É., Labelle, H. and de Guise, J. (1999) "Towards the self-calibration of a multi-view radiographic imaging system for the $3 \mathrm{D}$ reconstruction of the human spine and rib cage", Int. J. Pattern Recognition Artif. Intell. 13(4), 761-779.

[15] Trochu, F. (1993) "A contouring program based on dual kriging interpolation", Eng. Comput. 9, 160-177.

[16] Aubin, C.E., Descrimes, J.L, Dansereau, J., Skalli, W., Lavaste, F. and Labelle, H. (1995) "Geometric modeling of the spine and thorax for the biomechanical analysis of scoliotic deformities using the finite element method." Ann. Chir. 49(8), 749-761 (in French).

[17] Oden, J.T. and Ripperger, E.A. (1981) Mechanics of Elastic Structures, 2nd Ed. (McGraw-Hill, New York), p 351.

[18] Stokes, I.A.F., Bigalow, L.C. and Moreland, M.S. (1986) "Measurement of axial rotation of vertebrae in scoliosis", Spine 11, 213-218.

[19] Aubin, C.É., Lobeau, D., Labelle, H., Godillon, A.P., Le Blanc, R. and Dansereau, J. (1999) "Planes of maximum deformity in the scoliotic spine", Research into Spinal Deformities II (IOS Press, Amsterdam) Vol. 59, pp 45-48.

[20] Stokes, I.A.F. and Gardner-Morse, M. (1993) "Three-dimensional simulation of Harrington distraction instrumentation for surgical correction of scoliosis", Spine 18, 2457-2464.

[21] Duke, K.K., Hill, D.L., Moreau, M.J., Mahood, J.K., Raso, V.J. and Budney, D.L. (1999) "Force measurement of the derotation manoeuvre", Research into Spinal Deformity 2 (IOS Press, Amsterdam) Vol. 59, pp. 109-112.

[22] Gayet, L.E., Pries, P., Hamcha, H., Clarac, J.P. and Texereau, J. (1998) "Biomechanical study and digital modeling of traction resistance in posterior thoracic implants", 7(4), 313-320.

[23] Delorme, S., Labelle, H., Poitras, B., Rivard, C.H., Coillard, C. and Dansereau, J. (2000) "Pre-, intra-, and postoperative threedimensional evaluation of adolescent idiopathic scoliosis", J. Spinal Disord. 13(2), 93-101. 